\title{
GENERALIZED PRODUCT THEOREMS FOR TORSION INVARIANTS WITH APPLICATIONS TO FLAT BUNDLES
}

\author{
BY DOUGLAS R. ANDERSON ${ }^{1}$
}

Communicated by E. H. Spanier, October 13, 1971

This note announces generalizations of the product theorems for Wall invariants and Whitehead torsions due to Gersten [5], Siebenmann [7, Chapter VII], and Kwun and Szczarba [6], and applies these theorems to study torsion invariants of the total space of a flat bundle. The generalized product theorems are described in $\S \S 1$ and 2 . The applications are found in $\$ 3$.

These theorems were discovered in an attempt to understand more clearly the orientation phenomena discovered in [1] and [2] by concentrating attention on bundles in which "orientation" is a complete bundle invariant. The author would like to thank D. Sullivan whose use of the word "flat" in a private conversation stimulated this work.

0 . Basic algebraic definitions and notations. Let $R$ be a commutative ring with unit. (Usually $R=Z$, the ring of integers, or $Q$ the rational numbers.) For any group $\pi, \mathfrak{P R}(\pi)$, and $\sum \mathfrak{P R}(\pi)$ will denote the category of finitely generated projective modules over $R(\pi)$, and the category with objects $(P, f)$ with $P \in \mathfrak{P R}(\pi)$ and $f: P \rightarrow P$ and $R(\pi)$ isomorphism. A morphism $g:\left(P_{1}, f_{1}\right) \rightarrow\left(P_{2}, f_{2}\right)$ is an $R(\pi)$ homomorphism $g: P_{1} \rightarrow P_{2}$ such that $f_{2} g=g f_{1}$.

$K_{0} R(\pi)$ and $K_{1} R(\pi)$ will be usual algebraic $K$-theoretic groups (cf. [3, pp. 344-348]). [P] or [P,f] will denote the class of $P$ and $(P, f)$ in $K_{0} R \pi$ and $K_{1} R \pi$ respectively. The quotient of $K_{1} R(\pi)$ by the subgroup $\pm \pi$ will be denoted Wh $R(\pi)$ and will be called the $R$-Whitehead group of $\pi$. When $R=Z$ this is the usual Whitehead group. If $j: \pi \rightarrow \pi^{\prime}$ is a homomorphism, $j_{*}$ will denote any of the induced maps on $K_{0}, K_{1}$, or Wh.

Let $A$ and $B$ be groups and $\alpha: B \rightarrow$ Aut $A$ be a homomorphism. Then $A \times{ }_{\alpha} B$ will denote the semidirect product of $A$ and $B$ with respect to $\alpha$. As sets $A \times{ }_{a} B=A \times B$. The multiplication on $A \times{ }_{a} B$ is given by $(a, b)\left(a^{\prime}, b^{\prime}\right)$ $=\left(a \alpha(b)\left(a^{\prime}\right), b b^{\prime}\right)$. The functions $k: A \rightarrow A \times{ }_{\alpha} B, p: A \times{ }_{a} B \rightarrow B$, and $s: B$ $\rightarrow A \times{ }_{\alpha} B$ given by $k(a)=(a, 1), p(a, b)=b$, and $s(b)=(1, b)$ are homomorphisms. $\alpha$ extends to a homomorphism, also denoted by $\alpha, \alpha: B$ $\rightarrow$ Aut $R(A)$.

AMS 1970 subject classifications. Primary 57C10; Secondary 18F25.

Key words and phrases. Whitehead torsion, Wall invariant, flat bundle.

${ }^{1}$ Partially supported by the NSF under grant number GP-29540. 
1. $\alpha$-semilinear representations. Let $A, B, \alpha$ be as above and $M \in \mathfrak{P R}(A)$. A representation $\rho: B \rightarrow A^{-1 t_{R} M}$ is $\alpha$-semilinear (cf. [4]) if $\rho(b)(\lambda m)$ $=\alpha(b)(\lambda) \rho(b)(m)$ for all $b \in B, \lambda \in R(A), m \in M$.

Let $\mathfrak{R}(R(B), R(A), \alpha)$ be the category with objects pairs $(P, \rho)$ where $P \in \mathfrak{B R}(A)$ and $\rho: B \rightarrow \mathrm{Aut}_{R} P$ is $\alpha$-semilinear. A morphism $f:\left(P_{1}, \rho_{1}\right)$ $\rightarrow\left(P_{2}, \rho_{2}\right)$ in $\Re(R(B), R(A) \alpha)$ is an $R(A)$ map $f: P_{1} \rightarrow P_{2}$ such that $f \rho_{1}(b)$ $=\rho_{2}(b) f$ for every $b \in B$. The Grothendieck group of the category $\Re(R(B), R(A), \alpha)$ is called the group of $\alpha$-semilinear representations of $B$ over $R(A)$ and is denoted $G_{R}(B, A, \alpha)$. When $A=1, G_{R}(B, A, \alpha)=G_{R}(B)$ is the usual group of representations of $B$ over $R$.

THEOREM 1. There are pairings

$$
\begin{aligned}
& T_{0}: G_{R}(B, A, \alpha) \otimes K_{0} R(B) \rightarrow K_{0} R\left(A \times{ }_{\alpha} B\right), \\
& T_{1}: G_{R}(B, A, \alpha) \otimes K_{1} R(B) \rightarrow K_{1} R\left(A \times{ }_{\alpha} B\right) .
\end{aligned}
$$

SKETCH OF THE PROOF. The proof uses some ideas of Swan developed in [3, pp. 563-566]; namely, for $T_{0}$, define a functor

$$
\mathfrak{I}_{0}: \mathfrak{R}(R(B), R(A), \alpha) \times \mathfrak{P R}(B) \rightarrow \mathfrak{P R}\left(A \times{ }_{\alpha} B\right)
$$

by setting $\mathfrak{I}_{0}((P, \rho), Q)=P \otimes_{R} Q$ and making $P \otimes_{R} Q$ into an $R\left(A \times{ }_{\alpha} B\right)$ module by setting $(a, b) p \otimes q=a \rho(b)(p) \otimes b q$. By Frobenius reciprocity [3, p. 563], if $P$ is free over $R(A)$ of rank $m$ and $Q$ is free over $R(B)$ of rank $n$, then $P \otimes_{R} Q$ is free of rank $m n$ over $R\left(A \times{ }_{\alpha} B\right)$. A direct sum argument then shows that if $P \in \mathfrak{P R}(A)$ and $Q \in \mathfrak{P} R(B)$, then $P \otimes_{R} Q \in \mathfrak{B} R\left(A \times{ }_{\alpha} B\right)$. The proof is completed by noting that $\mathfrak{T}_{0}$ preserves short exact sequences in either variable.

The pairing $T_{1}$ is induced from the functor

$$
\mathfrak{T}_{1}: \mathfrak{R}(R(B), R(A), \alpha) \times \sum \mathfrak{P R}(B) \rightarrow \sum \mathfrak{P R}\left(A \times{ }_{\alpha} B\right),
$$

given by $\mathfrak{I}_{1}((P, \rho),(Q, f))=\left(P \otimes_{R} Q, 1 \otimes f\right)$, by using the categorical definition of $K_{1}$ given in [3, p. 348].

When $A=1$, the pairings $T_{0}$ and $T_{1}$ reduce to the standard module structure of $G_{R}(B)$ on $K_{0} R(B)$ and $K_{1} R(B)$ respectively (cf. [3, p. 565]).

Let $P$ be free over $R(A)$ of finite rank and $\rho: B \rightarrow$ Aut $_{R} P$ be $\alpha$-semilinear. By picking a basis $e_{1}, \ldots, e_{m}$ for $P$ and setting $\rho(b)\left(e_{i}\right)=\sum_{j} \beta_{i j} e_{j}$, we obtain for each $b \in B$ a nonsingular matrix, $\left(\beta_{i j}\right) \in \mathrm{GL}(m ; R(A))$, whose transpose is called the matrix of $\rho(b)$ with respect to $e_{1}, \ldots, e_{m} . \rho$ is simple if for all $b \in B$ the class of $\left(k\left(\beta_{i j}\right)\right)^{t}$ is zero in Wh $R\left(A \times{ }_{\alpha} B\right)$, where $k: R(A) \rightarrow$ $R\left(A \times{ }_{\alpha} B\right)$. This is independent of the choice of basis. Let $S_{R}(B, A, \alpha)$ $\subset G_{R}(B, A, \alpha)$ be the subgroup generated by the simple representations. Extending Theorem 1, we have

LEMMA 2. $T_{1}$ induces a pairing $T_{1}^{\prime}: S_{R}(B, A, \alpha) \otimes W h R(B) \rightarrow W h R\left(A \times{ }_{\alpha} B\right)$. 
Proof. For any $b \in B$, let $f_{b}: R(B) \rightarrow R(B)$ be the $R(B)$ linear map that sends 1 to $b$. By giving the pairing $T_{1}$ a matrix interpretation as in the proof of [1, Propositon 1.1], one verifies that if $\rho: B \rightarrow \mathrm{Aut}_{R} P$ is simple, then $T_{1}\left([P, \rho] \otimes\left[R(B), f_{b}\right]\right)=\left[P \otimes_{R} R(B), 1 \otimes f_{b}\right]=0$ in Wh $R\left(A \times{ }_{\alpha} B\right)$. Since Wh $R(B)=K_{1} R(B) /\left(\right.$ subgroup generated by $\pm\left[R(B), f_{b}\right]$ ) where $b \in B$, the lemma follows.

The pairings $T_{0}, T_{1}$, and $T_{1}^{\prime}$ are natural in a sense we now make precise.

Let $C$ be a group and $\gamma: B \rightarrow$ Aut $C$. A homomorphism $\sigma: A \rightarrow C$ is admissible if $\sigma(\alpha(b)(a))=\gamma(b) \sigma(a)$ for all $a \in A, b \in B$. If $\sigma: A \rightarrow C$ is admissible, then $\sigma \times 1: A \times{ }_{\alpha} B \rightarrow C \times{ }_{\gamma} B$ is a homomorphism. An admissible homomorphism $\sigma: A \rightarrow C$ induces a homomorphism $\sigma_{*}: G_{R}(B, A, \alpha)$ $\rightarrow G_{R}(B, C, \gamma)$ by setting $\sigma_{*}[P, \rho]=\left[R(C) \otimes_{R(A)} P, \gamma \otimes \rho\right]$ where $R(C)$ becomes a right $R(A)$ module via $\sigma$.

TheOREM 3. Let $\sigma: A \rightarrow C$ be admissible. Then

$$
(\sigma \times 1)_{*} T_{i}=T_{i}\left(\sigma_{*} \times 1\right) \quad \text { for } i=0,1
$$

and

$$
(\sigma \times 1)_{*} T_{1}^{\prime}=T_{1}^{\prime}\left(\sigma_{*} \times 1\right) .
$$

COROllary 4. The usual module structure $T: G_{R}(B) \otimes K_{i} R(B) \rightarrow K_{i} R(B)$ is a direct summand of $T_{i}: G_{R}(B, A, \alpha) \otimes K_{i} R(B) \rightarrow K_{i} R\left(A \times{ }_{\alpha} B\right), i=0,1$.

Proof. Apply Theorem 3 to the admissible homomorphisms $\sigma: A \rightarrow 1$ and $\tau: 1 \rightarrow A$ where 1 is the trivial group.

2. The generalized product theorems. Let $C_{*}$ be a finitely generated chain complex of projectives over $R(A)$ and $\rho: B \rightarrow \mathrm{Aut}_{R}\left(C_{*}\right)$ be an $\alpha$-semilinear representation such that for each $b \in B, \rho(b)$ is a chain map. Let $\rho_{i}: B$ $\rightarrow \operatorname{Aut}_{R}\left(C_{i}\right)$ denote the restriction of $\rho$ to $C_{i}$. We set

$$
\chi\left(C_{*}, \rho\right)=\sum(-1)^{i}\left[C_{i}, \rho_{i}\right] \in G_{R}(B, A, \alpha)
$$

and call $\chi\left(C_{*}, \rho\right)$ the Euler characteristic of $\rho$.

If $D_{*}$ is a chain complex over $R(B)$, the proof of Theorem 1 shows that the usual tensor product of chain complexes $C_{*} \otimes_{R} D_{*}$ is a chain complex over $R\left(A \times{ }_{\alpha} B\right)$.

THEOREM 5. Let $C_{*}, \rho$, and $D_{*}$ be as above and suppose that $D_{*}$ is dominated by a finitely generated chain complex of projectives over $R(B)$. Then $C_{*} \otimes_{R} D_{*}$ is dominated by a finitely generated chain complex of projectives over $R\left(A \times{ }_{\alpha} B\right)$ and

$$
w\left(C_{*} \otimes_{R} D_{*}\right)=\chi\left(C_{*}, \rho\right) w\left(D_{*}\right)
$$

where $w$ denotes the Wall invariant and juxtaposition denotes the pairing $T_{0}$. 
THEOREM 6. Let $C_{*}$ be a finitely generated, based chain complex over $R(A)$ and let $\rho: B \rightarrow A u t_{R}\left(C_{*}\right)$ be an $\alpha$-semilinear representation such that for each $i, \rho_{i}$ is simple. Let $D_{*}$ be a finitely generated, based, acyclic chain complex over $R(B)$. Then $C_{*} \otimes_{R} D_{*}$ is a finitely generated, based, acyclic chain complex over $R\left(A \times{ }_{\alpha} B\right)$ and

$$
\tau_{R}\left(C_{*} \otimes_{R} D_{*}\right)=\chi\left(C_{*}, \rho\right) \tau_{R}\left(D_{*}\right)
$$

where $\tau_{R}$ denotes $R$-Whitehead torsion and juxtaposition denotes the pairing $T_{1}^{\prime}$.

These theorems are obtained by an induction argument similar to that of $[6, p .188]$.

Since $\rho(b)$ is a chain map for each $b \in B$, there are induced $\alpha$-semilinear representations $\bar{\rho}_{i}: B \rightarrow \operatorname{Aut}_{R}\left(H_{i}\left(C_{*}\right)\right)$. If $H_{i}\left(C_{*}\right) \in \mathfrak{B} R(A)$ for all $i$, we set $\chi\left(H_{*}\left(C_{*}\right), \bar{\rho}\right)=\sum(-1)^{i}\left[H_{i}\left(C_{*}\right), \bar{\rho}_{i}\right] \in G_{R}(B, A, \alpha)$.

LEMMA 7. Let $C_{*}$ be a finitely generated chain complex of projectives over $R(A)$ such that $H_{i}\left(C_{*}\right) \in \mathfrak{P R}(A)$ for all $i$ and let $\rho: B \rightarrow \operatorname{Aut}_{R}\left(C_{*}\right)$ be an $\alpha$-semilinear representation as above. Then $\chi\left(C_{*}, \rho\right)=\chi\left(H_{*}\left(C_{*}\right), \bar{\rho}\right)$.

3. Flat bundles. Let $\xi=(E, p, B, F)$ be a PL fiber bundle (cf. [1]) and $\pi=\pi_{1}\left(B, b_{0}\right) . \xi$ is flat if there is a triangulation $K$ of $F$ with vertex $v$, and a homomorphism $\omega: \pi \rightarrow \operatorname{Iso}(K, v)$, the simplicial isomorphism of $K$ leaving $v$ fixed, such that $\xi=\left(\widetilde{B} \times{ }_{\pi} F, p^{\prime}, B, F\right)$ where $\widetilde{B}$ is the universal cover of $B, \beta \in \pi$ acts on $\widetilde{B} \times F$ by $\beta(x, y)=(\beta x, \omega(\beta) y)$, and $p^{\prime}: \widetilde{B} \times{ }_{\pi} F \rightarrow B$ is the natural map. $\xi$ is called the flat bundle associated with $\omega: \pi \rightarrow \operatorname{Iso}(K, v)$.

Let $s: B \rightarrow E$ be the natural cross section $s(b)=v_{b}$ where $v_{b}$ is the image of $v$ in $p^{-1}(b)$ and let $e_{0}=s\left(b_{0}\right)$. Then $s_{*}$ splits the homotopy exact sequence of $\xi$. Furthermore

Lemma 8. Define $\alpha: \pi_{1}\left(B, b_{0}\right) \rightarrow$ Aut $\pi_{1}(F, v)$ by $\alpha(\beta)=\omega(\beta)_{*}: \pi_{1}(F, v)$ $\rightarrow \pi_{1}(F, v)$. Then $i_{*} \times s_{*}: \pi_{1}(F, v) \times{ }_{\alpha} \pi_{1}\left(B, b_{0}\right) \rightarrow \pi_{1}\left(E, e_{0}\right)$ is an isomorphism.

In the remainder of this section we identify these two groups via this isomorphism.

Let $\xi$ be the flat bundle associated with $\omega: \pi \rightarrow \operatorname{Iso}(K, v)$. Let $\widetilde{K}$ be the triangulation of $\widetilde{F}$, the universal cover of $F$, covering $K$ and $C_{*}(\tilde{K} ; R)$ be the cellular chains on $\widetilde{K}$ with $R$ coefficients viewed as a (based) $R \pi_{1}(F, v)$ module (when necessary). Since each $f \in \operatorname{Iso}(K, v)$ fixes $v, f$ is covered by a simplicial isomorphism $\tilde{f}:(\widetilde{K}, \tilde{v}) \rightarrow(\widetilde{K}, \tilde{v})$ where $\tilde{v}$ is a fixed vertex of $\widetilde{K}$ over $v$. The correspondence $f \rightarrow \vec{f}$ defines a homomorphism $\lambda: \operatorname{Iso}(K, v)$ $\rightarrow \operatorname{Iso}(\tilde{K}, \tilde{v})$. Define $\rho: \pi_{1}\left(B, b_{0}\right) \rightarrow \operatorname{Aut}_{R}\left(C_{*}(\tilde{K} ; R)\right)$ by

$$
\rho(\beta)=\lambda \omega(\beta)_{*}: C_{*}(\tilde{R} ; R) \rightarrow C_{*}(\tilde{R} ; R) .
$$


Relative to the $\alpha$ of Lemma $8, \rho$ is $\alpha$-semilinear.

ThEOREM 9. Let $\xi=(E, p, B, F)$ be the flat bundle associated with $\omega: \pi_{1}\left(B, b_{0}\right) \rightarrow \operatorname{Iso}(K, v)$ and suppose $B$ is dominated by a finite complex and $F$ is compact. Then $E$ is dominated by a finite complex and

$$
w(E)=\chi\left(C_{*}(\tilde{K} ; Z), \rho\right) w(B)
$$

where $w$ denotes the (unreduced) Wall invariant.

THEOREM 10. Let $\xi=(E, p, B, F)$ be the flat bundle associated with $\omega: \pi_{1}\left(B, b_{0}\right) \rightarrow \operatorname{Iso}(K, v)$ and suppose that the subcomplex $A$ is a deformation retract of $B$. Then $E_{A}=p^{-1}(A)$ is a deformation retract of $E$ and

$$
\tau_{R}\left(E, E_{A}\right)=\chi\left(C_{*}(\tilde{K} ; R), \rho\right) \tau_{R}(B, A)
$$

where $\tau_{R}$ denotes Whitehead torsion over $R$.

The idea of the proof of these theorems is to note that the universal cover $\tilde{E}$ of $E$ is $\tilde{F} \times \tilde{B}$, that $(\beta, \gamma) \in \pi_{1}(F, v) \times{ }_{\alpha} \pi_{1}\left(B, b_{0}\right)$ acts by $(\beta, \gamma)(x, y)$ $=(\beta \lambda \omega(\gamma) x, \gamma y)$, and, therefore, that $C_{*}(\tilde{E} ; R) \approx C_{*}(\tilde{F} ; R) \otimes_{R} C_{*}(\tilde{B} ; R)$ as modules over $\pi_{1}\left(E, e_{0}\right)=\pi_{1}(F, v) \times_{\alpha} \pi_{1}\left(B, b_{0}\right)$. The proof is completed by applying Theorems 5 and 6 , respectively.

Suppose now that $\pi_{1}(F, v)$ is finite. Then $\tilde{K}$ is a finite complex and the $Q \pi_{1}(F, v)$ module $H_{*}(\widetilde{K} ; Q)$ is finitely generated over $Q$. By Maschke's Theorem [3, p. 559], $H_{*}(\widetilde{K} ; Q)$ and $H_{i}(\widetilde{K} ; Q)$ are finitely generated projectives over $Q \pi_{1}(F, v)$. Hence $\chi\left(C_{*}(\tilde{K} ; Q), \bar{\rho}\right)=\chi\left(H_{*}(\tilde{K} ; Q), \bar{\rho}\right)$ by Lemma 7. Since the representation $\bar{\rho}: \pi_{1}\left(B, b_{0}\right) \rightarrow \operatorname{Aut}_{Q}\left(H_{*}(\tilde{R} ; Q)\right)$ depends only on the structure $\xi$ as a bundle with structure group $\operatorname{Homeo}(F, v)$ (i.e., $\rho$ depends only on topological properties of the bundle $\xi$ ), we have the

Corollary 11. Let $\xi=(E, p, B, F)$ and $A \subset B$ be as in Theorem 10. If $\pi_{1}(F, v)$ is finite, then the rational Whitehead torsion, $\tau_{Q}\left(E, E_{A}\right)$, depends only on $\tau(B, A)$ and the topological structure of the bundle $\xi$.

\section{BIBLIOGRAPHY}

1. D. R. Anderson, The Whitehead torsion of the total space of a fiber bundle, Topology (to appear).

2. Wall's finiteness obstruction for the total space of a flat bundle (submitted).

3. H. Bass, Algebraic K-theory, Benjamin, New York, 1968. MR 40 \#2736.

4. F. T. Farrell and W. C. Hsiang, $A$ formula for $K_{1} R_{a}[T]$, Applications of Categorical Algebra, Proc. Sympos. Pure Math., vol. 17, Amer. Math. Soc., Providence, R.I., 1970, pp. 192-218. MR 41 \# 5457.

5. S. M. Gersten, A product formula for Wall's obstruction, Amer. J. Math. 88 (1966), 337-346. MR 33 \#6623.

6. K. W. Kwun and R. H. Szczarba, Product and sum theorems for Whitehead torsion, Ann. of Math. (2) 82 (1965), 183-190. MR $32 \# 454$.

7. L. C. Siebenmann, The obstruction to finding a boundary for an open manifold of dimension greater than five, Thesis, Princeton University, Princeton, N.J., 1965.

Department of Mathematics, Syracuse University, Syracuse, New York 13210 